\title{
Aproximación al ejercicio de la ciudadanía plena por parte del cuerpo de oficiales de las Fuerzas Militares colombianas*
}

\section{Approach to the exercise of full citizenship by the officer corps of the Colombian Military Forces}

Diego Nicolás Fonseca Moreno

\section{Resumen}

Este texto tratará la relación entre el ejercicio de la ciudadanía del cuerpo de oficiales y los derechos económicos y sociales, haciendo hincapié en el derecho a la educación, dado su importante papel, así como su ejercicio condicionado debido al mal desarrollo de los conocimientos y habilidades cívicas; por lo tanto, se proporciona una breve consideración sobre el significado de "ser ciudadano", así como una auto representación basada en una "ciudadanía paralela".

\section{Palabras Clave}

Colombia; fuerzas militares; ciudadanía; derechos económicos y sociales.

Artículo de Reflexión.

** Politólogo con estudios en Gobierno, Gerencia y Asuntos Públicos, auditor interno en la ISO 37001 (sistema de gestión antisoborno) y estudiante de la Especialización en Derechos Humanos y Competencias Ciudadanas (Universidad del Tolima). Con interés en el análisis socio-jurídico, las políticas públicas y las políticas TIC. Correo electrónico: dnfonseca@ academia.usbbog.edu.co 


\section{Abstract}

This text will deal with the relationship between exercise of citizenship and socio-economic rights, while emphasizing on the right to education given its important role as well as its conditioned implementation due to poor development of civic knowledge and skills; therefore a short consideration about the meaning of "being a citizen" is provided as well as a self-representation based on a "parallel citizenship".

\section{Key Words}

Colombia; military forces; citizenship; economic and social rights.

\section{Introducción}

Se debe tener en cuenta que Colombia ha tenido un conflicto armado interno bastante extenso, doloroso y lleno de errores, donde la sociedad civil ha sido la más perjudicada. En este, las Fuerzas Militares (FF.MM) han sido uno de los actores estatales que más ha ocupado recursos, además, ha contado con una opinión altamente favorable por parte de la población.

Dicha opinión ha sido medida mediante sondeos realizados por diversas encuestadoras, en estos se observan diversos porcentajes que las hacía acreedoras a uno de los mayores niveles de favorabilidad entre las instituciones estatales, pero esto cambió en el año 2020. De acuerdo con el portal La Silla Vacía (2020), "por primera vez en 15 años, hay tanta gente con una imagen favorable como desfavorable de las Fuerzas Militares," por lo cual, han dejado de gozar de una percepción positiva casi unánime por parte de la ciudadanía. 
Al tener estos cambios en su imagen favorable surge la necesidad de aproximarse a este grupo de la población, sin embargo, Eduardo Pizarro (2019), quién se ha dedicado a estudiar el ámbito militar, destaca que ha sido casi nulo el análisis micro acerca de las percepciones de sus miembros sobre sí mismos y sobre su papel en la sociedad. por lo que genera un incentivo a tender puentes que permitan transformaciones positivas en el ámbito militar.

Esta limitación se acentúa aún más si se trata sobre el ejercicio de la ciudadanía al interior de las Fuerzas Militares, en cuanto el concepto de la ciudadanía suele asociarse al veto que tienen sus derechos políticos y a una serie de limitaciones en sus derechos civiles, sumado al escaso material académico que indaga esta temática.

Para aportar al estado de la cuestión, este texto de reflexión tiene el objetivo de indagar y analizar sobre el ejercicio de la ciudadanía en el cuerpo de oficiales, a partir del acceso a la educación, por ser tanto un derecho económico, social y cultural, también hace parte de los sistemas de promoción, es uno de los principios del Sistema Educativo (equidad y acceso a la educación) y un elemento para consolidar la capacidad de autorregulación.

Para ello, se hará un recuento sobre los derechos humanos, en especial los Derechos Económicos, Sociales y Culturales (DESC), destacando su importancia y la dinámica alrededor de su exigibilidad, luego presentará la perspectiva analítica en la sección de la idea de ciudadanía y su ejercicio, siendo esta una aproximación y cierra con el apartado de las conclusiones.

Por otra parte, este artículo se elaboró bajo el paradigma cualitativo y el enfoque hermenéutico. Como técnicas de recolección y de análisis de información se utilizó el método documental y descriptivo; el primero, por la consulta realizada a diversos documentos como artículos de revistas indexadas y periódicos, páginas de internet, y datos 
almacenados por diversas entidades. El segundo, en cuanto se plantea un análisis del ejercicio de la ciudadanía por parte del cuerpo de oficiales de las Fuerzas Militares colombianas.

\section{De los derechos humanos y el grupo de los económicos, sociales y culturales}

Al hablar de los derechos humanos, inicialmente se destaca que no son fácilmente percibidos y reconocidos en la vida cotidiana ${ }^{1}{ }^{2}$, sin embargo, son de vital importancia, por ser el conjunto de derechos que nos corresponden a todas las personas sin ninguna distinción y están concebidos para que no pueden suprimirse, aunque sí pueden limitarse en situaciones excepcionales y de manera claramente determinadas.

La evolución del concepto de derechos humanos está ligada a los procesos históricos del mundo occidental, por lo cual ha contado con tres momentos donde se han conquistado: Un primer momento corresponde al siglo XVIII, donde surgió la construcción del Estado Liberal, además de la conquista de los derechos civiles, ligados a la libertad individual. El segundo se ubica en el siglo XIX en el que se consolidan los derechos políticos que permiten ejercer la posibilidad de participar en el ejercicio del poder político.

Y el tercer momento se puede identificar en el siglo XX, donde se materializaron medidas de seguridad y bienestar desde lo económico, en otras palabras, surgen los Derechos Económicos, Sociales y Culturales, que tratan sobre el acceso a la alimentación, a la vivienda

1 Sería pertinente poder contar con ejercicios cuantitativos sobre la percepción y el reconocimiento de los derechos humanos en la cotidianidad.

2 De igual manera, se destaca que, en América Latina, se suele asociar los derechos humanos a la exigibilidad ante las entidades estatales para enfrentar hechos como la tortura o la desaparición forzada, al ser estas las violaciones brutales de los derechos más elementales de las personas (Instituto Interamericano de Derechos Humanos, 2010). 
adecuada, a la educación, a la salud, a la seguridad social, a la participación en la vida cultural, al agua y saneamiento, y al trabajo.

En este mismo siglo XX, se avanzó en la consagración de estos ideales mediante la firma de la Declaración Universal de Derechos Humanos junto con la aprobación de dos Pactos: uno sobre los Derechos Civiles y Políticos (1966) y el otro sobre Derechos Sociales, Económicos y Culturales (1966). A partir de la década de los ochenta, en medio de una crisis económica, surgió una serie de tendencias y acontecimientos políticos y sociales orientados a la protección de los derechos humanos, entre ellos está la acogida del principio de integralidad.

Este principio consiste, según García (2003), en que todos los derechos humanos se reclaman, condicionan y perfeccionan mutuamente, de modo que es necesario protegerlos y darles a todos la misma atención. Sin embargo, este mismo autor recalca que el avance en la integralidad de los derechos humanos se ha reconocido más en el plano conceptual y normativo, que en el procesal u operativo.

Este devenir histórico permitió que en Colombia se reconociera e incorporara en el capítulo II de la Constitución Política de 1991 estos derechos, así mismo, se avanzó en su exigibilidad, a pesar de que no se desencadena automáticamente un reclamo concreto en forma inmediata, como si puede suceder con los derechos civiles y políticos.

Desde la jurisprudencia de la Corte Constitucional se ha transformado la concepción de estos derechos, pues pasan de ser derechos por conexidad a ser disposiciones fundamentales, que merecen protección reforzada autónoma por mandato de la Constitución (2010, pág. 42). Todo esto se materializa con la creación de jurisprudencia desde la rama judicial, por medio de las Políticas Públicas, además de diversas acciones gubernamentales y de la sociedad civil. 
En el siguiente apartado se destacarán los documentos orientadores de su quehacer misional y su relación con el ejercicio de la ciudadanía, tanto para el cuerpo de oficiales como para el resto de la población.

\section{Las Fuerzas Militares: Su papel, relación y sistema de educación}

En primera instancia, la Constitución Política, ha definido como finalidad primordial de las Fuerzas Militares "la defensa de la soberania, la independencia, la integridad del territorio nacional y del orden constitucional (Constitución Política de Colombia, 1991, Artículo 217).”

Para acatar dicha finalidad, entre el siglo XX y XXI se ha desarrollado una relación entre el poder militar y el civil donde se reconoce la supremacía del último sobre el primero, sin embargo, esto no ha estado exento de tensiones, dado que:

(...) los civiles no están adecuadamente involucrados y formados en los temas relacionados con la seguridad y la defensa, lo cual deja un margen de autonomía para el estamento castrense en lo referente al diseño y conducción de las políticas en el sector (Andrade Becerra, 2012, pág. 164).

Como muestra de esto, Rodríguez Gómez (2020) expone que las doctrinas de seguridad en sus diferentes versiones identificaron las acciones de las Fuerzas Militares con medidas coercitivas que se movieron al ritmo de las alteraciones del orden, sin garantizar a cambio la concomitante protección de las libertades y los derechos (págs. 113-115).

Todo esto ha generado un contexto donde el Estado y las Fuerzas Militares mantengan unas relaciones distantes con la población, pero uno de los mayores niveles de favorabilidad entre las instituciones estatales, generando un ejercicio particular de la ciudadanía en Colombia. 
Dicho ejercicio está enmarcado en dos elementos fundamentales: la Política Pública y el Sistema Educativo de las Fuerzas Armadas-SEFA ${ }^{3}$; sobre la primera, desde el 2019 se definió la Política de Defensa y Seguridad-PDS para la Legalidad, el Emprendimiento y la Equidad; en esta se plantean las amenazas, un apartado conceptual, los ejes de la transformación estratégica, principios de la Política, su propósito, junto con los objetivos estratégicos y las líneas de Política (Ministerio de Defensa Nacional, 2019).

En cuanto al Sistema Educativo, este se origina por la redefinición del Proyecto Educativo de las Fuerzas Armadas-PEFA-4, por ello, desde el Ministerio de Defensa Nacional se concibió a este sistema SEFA define como "el conjunto de elementos interrelacionados en un circulo virtuoso para formar, actualizar, capacitar, instruir y entrenar a los integrantes de las fuerzas armadas (2008, pág. 13)”.

En este mismo sentido, dentro del Plan Estratégico del Sistema Educativo de las Fuerzas Armadas PESE 2007-20195 se incluyen los principios y valores del SEFA, a saber: calidad educativa, pertinencia, enfoque humanista y el ya mencionado, equidad y acceso a la educación. Así mismo, a sus cuatro subsistemas: gestión de Doctrina, aseguración de la calidad educativa, certificación militar, y de investigación, desarrollo e innovación (Ministerio de Defensa Nacional, 2008, págs. 17-19).

3 Aquí es curioso que concibieran este sistema educativo para las fuerzas armadas, siendo que la Constitución Política habla de las Fuerzas Militares, no armadas.

4 El Proyecto Educativo de las Fuerzas Armadas (PEFA) precisa los principios filosóficos, antropológicos, pedagógicos y didácticos de la formación del soldado; inspira la visión y misión del Sistema Educativo de las Fuerzas Armadas (SEFA) y los propósitos e intencionalidades sociales, militares y culturales (Ramírez Rubiano \& Ramírez Bravo, 2015).

5 Dicho plan contiene los principios y valores, las políticas educativas, la misión y visión del SEFA, el mapa de procesos, la oferta de valor y capacidades distintivas, los objetivos del sistema educativo y el seguimiento y evaluación a estas acciones (Ministerio de Defensa Nacional, 2008). 
En síntesis, las Fuerzas Militares han contado con autonomía para el diseño y conducción de las políticas, los civiles no están adecuadamente involucrados, dado que identifican su quehacer con medidas coercitivas, sin garantizar a cambio la concomitante protección de las libertades y los derechos.

Además, poseen un sistema educativo donde se plantea una interrelación de sus elementos, pero no hay claridad sobre esta interrelación para orientar las Políticas Públicas de educación, seguridad y defensa, al ser estas últimas, sistemas complejos de decisión y control ${ }^{6}$.

Todo lo aquí planteado nos permite destacar que la evolución del concepto de derechos humanos está incluida en los documentos orientadores de su quehacer misional, teniendo claro las diferencias que se pueden presentar entre lo planteado y lo implementado. Por ello, el siguiente apartado se ocupará de analizar su ejercicio de la ciudadanía, teniendo como referencia su papel en la sociedad.

\section{La idea de ciudadanía y su ejercicio}

Al tener un soporte conceptual frente a los derechos humanos y la particularidad de las Fuerzas Militares, se estima pertinente partir de dos elementos para investigar el ejercicio de la ciudadanía plena. El primero, seguir la sugerencia de Marshall sobre la ampliación del concepto de ciudadanía, planteando que esta:

no debía quedar limitada a la titularidad de los derechos políticos, sino que debía comprender una dimensión social que permitiera el disfrute efectivo de los derechos y las garantías sociales, económicas y culturales (Pérez Luño, 1989; citado por Giraldo-Zuluaga, 2015, pág. 87)

6 Teniendo en cuenta la diversidad de definiciones de Política Pública, Ios elementos aquí planteados son compartidos. 
En segundo lugar, retomar lo destacado por Giraldo-Zuluaga (2015, pág. 87), quién cita de Año (2002), los elementos de una ciudadanía plena, a saber: contar con un trabajo digno y ejercer los derechos sociales y económicos. Así mismo, priorizar su construcción como la realización efectiva de una forma de vida y de convivencia entre los seres humanos en sociedad (2015, pág. 88).

Por lo anterior, la educación toma importancia, por ser parte de los fundamentos para avanzar hacia la garantía de una calidad de vida y el bienestar de la sociedad ${ }^{7}$ y, siguiendo a Nussbaum (2014), "no solo prepara para la ciudadanía, sino también para el trabajo y, sobre todo, para darle sentido a muestra vida (pág. 28).”

Teniendo en cuenta que la Constitución Política establece la finalidad y orienta el quehacer de las Fuerzas Militares, se debe tener en cuenta el artículo 222, que dice:

La ley determinará los sistemas de promoción profesional, cultural y social de los miembros de la Fuerza Pública. En las etapas de su formación, se les impartirá la enseñanza de los fundamentos de la democracia y de los derechos humanos (Constitución Política de Colombia, 1991, Artículo 222)

En esta orientación se observa que, a primera vista, la ciudadanía no está incluida dentro de esta disposición constitucional sobre la formación. Dicha exclusión se materializa con el poco énfasis que se hace en el tema de la ciudadanía en los programas de formación militar (Rivera Paez, 2019, pág. 220).

Por otra parte, desde la Política de Defensa y Seguridad-PDS, el concepto de ciudadanía está ligado a su ejercicio (en términos generales y sin diferenciar entre civiles y militares), además como un fundamento para la construcción de la cultura de la legalidad, así

7 Se debe destacar que es una expresión de la idea humanista de progreso, y se podría decir que están dentro de los principios y valores del SEFA. 
mismo, es mencionado en las líneas de política sobre la comunicación directa y transparente.

Sin embargo, es pertinente plantearse como temática central a la educación y su acceso, por ser tanto un derecho económico, social y cultural de acuerdo con el Capitulo II de la Constitución y hace parte de los sistemas de promoción. Además, es uno de los principios del Sistema Educativo (equidad y acceso a la educación) y un elemento para consolidar la capacidad de autorregulación en función de una conciencia ética, según el Plan Estratégico del Sistema Educativo de las Fuerzas Armadas.

Se puede destacar que el acceso a la educación por parte del cuerpo de oficiales de las Fuerzas Militares adquiere notoriedad por estar dentro de un sector selecto de la sociedad con oportunidades para acceder a la educación, por lo siguiente: De acuerdo con los datos obtenidos en la encuesta realizada por Samuel Rivera (2019):

el 2,3\% de los oficiales ha alcanzado como máximo nivel de educación el bachillerato, el 1,3\% algún nivel tecnológico, el 46,6 \% cuenta con un título profesional y el 49,7 \% ostenta estudios de posgrado $(38,9 \%$ de especialización, 10,6 \% de maestría y 0,2 \% de doctorado) (pág. 127).

Al revisar los porcentajes, destacan los altos porcentajes de títulos profesionales y de posgrado dentro del cuerpo de oficiales, esto es producto de las reformas en donde está incluida la Política de Consolidación de la Seguridad Democrática, que abordó el Proyecto de Modernización y Reestructuración de la educación de la Fuerza Pública.

Por otra parte, al concebir que la educación incide en la capacidad de autorregulación y en la proyección de un sentido de trascendencia individual, cabe la posibilidad de fortalecer las competencias para ser ciudadano en un sentido amplio; en poder participar en la 
forma como se construye y se convive en una comunidad, sin limitarse a un aprendizaje mecánico de ciertas normas (jurídicas, legales y políticas), para que los militares, como cualquier otro miembro de la sociedad, ejerzan su ciudadanía.

Estos condicionamientos en su ejercicio de la ciudadanía se expresan por las limitaciones en los modelos de enseñanza, donde se privilegia la jerarquización mediante el aprendizaje mecánico y no el dialógico, consolidando un déficit de las competencias ciudadanas, que se refleja en los bajos puntajes de los integrantes de las escuelas de formación militar en la sección de competencias ciudadanas en el examen Saber Pro ${ }^{8}$.

En otras palabras, al no hablarse de ciudadanía en la Constitución, ni en el Sistema Educativo ni en las escuelas, es plausible asumir que estos bajos niveles en el Saber Pro sean consecuencia de omitir este concepto en su formación y que, a causa de esto, el goce efectivo de los derechos de la sociedad se vea afectado. En últimas termina siendo la expresión del déficit en el entendimiento sobre el significado de ser ciudadano y emerge en la configuración de su identidad, una auto representación basada en una "ciudadanía paralela."

Esta auto representación se plantea a partir de su sentido de exclusividad y singularidad de la ciudadanía y del servicio público, que los hace sentirse un tipo diferente de ciudadano, con una mayor carga de obligaciones que de beneficios (Rivera Paez, 2019)9 ${ }^{9}$. Es plausible definir aquí que esto es producto del corto entendimiento sobre ser ciudadano y es atribuible a la carencia de recursos, la desigualdad de oportunidades y la estigmatización de los beneficiarios

8 En especial en el segundo semestre del 2014 y primero del 2015, de acuerdo con Rivera, (2019).

9 Para profundizar esto, puede revisar el libro Militares e identidad. Autorrepresentación y construcción de paz en el cuerpo de oficiales de las Fuerzas Militares colombianas. 
de asistencia social (Noya, 2002; citado por Giraldo-Zuluaga, 2015, pág. 87).

Aquí es pertinente retomar la sugerencia de Marshall sobre la ampliación del concepto de ciudadanía. Para eso es necesario debatir e incorporar el concepto de la ciudadanía en los programas de formación militar, además de las temáticas a reconocer por parte de la población civil relacionados con la educación de las Fuerzas Militares, la defensa y la seguridad nacional, sin que riña con la garantía del derecho al acceso a la información pública.

Así mismo, se debe determinar cuánto deben saber los militares acerca de la política, teniendo en cuenta la responsabilidad del Gobierno en educar a los militares en política, para que, a su vez, el jefe militar, comprenda la obligación de que, dentro de sus roles, se debe tener una dimensión política (Gramsci, 1999); citado por Rivera Paez, 2019). En últimas, conciban la concomitante protección de las libertades y los derechos.

A pesar de percibirse con una auto-representación basada en una "ciudadanía paralela", destacan la necesidad de mejorar el sistema de educación, como uno de los elementos para tener en cuenta para construir paz en Colombia (Rivera Paez, 2019). Con esta necesidad compartida, puede considerarse la educación como un tema para mejorar las relaciones entre civiles y militares y así empezar las transformaciones positivas que contribuyan al ejercicio pleno de la ciudadanía.

\section{Conclusión}

Se puede indagar sobre el ejercicio de una ciudadanía plena en los oficiales de las FF.MM a partir del ejercicio de uno de los derechos sociales y económicos, el de la educación. Para ello, se debe considerar que las Fuerzas Militares han contado con autonomía 
para el diseño y conducción de las políticas y los civiles no están adecuadamente involucrados, por cuanto identifican su quehacer con medidas coercitivas, además, en el artículo 222 de la Constitución Política, el concepto de ciudadanía no está incluida.

Por otra parte, poseen un sistema educativo que les permite un alto acceso a la educación, pero condiciona el ejercicio de la ciudadanía por la ausencia de orientaciones constitucionales y el déficit en el desarrollo de las competencias ciudadanas, lo que conlleva a una comprensión corta sobre el significado de ser ciudadano. Sin embargo, comparten la necesidad de mejorar el sistema de educación, por lo que puede considerarse la educación como un tema para mejorar las relaciones entre civiles y militares.

Con esta síntesis, se considera pertinente profundizar en el ejercicio de la ciudadanía en las Políticas Públicas de educación, seguridad y defensa, para luego indagar si el Sistema Educativo de las Fuerzas Armadas (SEFA) es promotor de competencias ciudadanas y así poder profundizar en el ejercicio de la ciudadanía y contribuir a la protección de las libertades y los derechos.

\section{Referencias}

Andrade, 0. (2012). Relaciones cívico-militares en Colombia: apuntes para un estado del arte. Análisis Internacional (Cesada a partir de 2015), 1(6). Recuperado de: https:// revistas.utadeo.edu.co/index.php/RAl/article/view/850/861

Constitución Política de Colombia (1991) Capitulo II de los derechos sociales, económicos y culturales.

Constitución Política de Colombia (1991) Capitulo VII de la Fuerza Pública.

García, S. (2003). Protección jurisdiccional internacional de los derechos económicos, sociales y culturales. Cuestiones Constitucionales(9), 127-157. Recuperado de: https:// docs.escr-net.org/usr_doc/CUC00905.pdf

Giraldo, G. A. (2015). Ciudadanía: aprendizaje de una forma de vida. Educ.Educ., 18(1). 
Instituto Interamericano de Derechos Humanos. (2010). Cartilla básica sobre derechos económicos, sociales y culturales. Recuperado de: https://www.iidh.ed.cr/IIDH/media/1465/cartilla-desc-2010.pdf

Ministerio de Defensa Nacional. (2008). Plan Estratégico del Sistema Educativo de las Fuerzas Armadas PESE 2007-2019. Recuperado de: https://www.mindefensa.gov.co/irj/ go/km/docs/Mindefensa/Documentos/descargas/estrategia_planeacion/desa_capital/Pagina/PESE_FINAL.pdf

Ministerio de Defensa Nacional. (2019). Política de Defensa y Seguridad-PDS para la Legalidad, el Emprendimiento y la Equidad. Recuperado de: https://www.mindefensa. gov.co/iri/go/km/docs/Mindefensa/Documentos/descargas/Prensa/Documentos/politica_defensa_deguridad2019.pdf

Nussbaum, M. C. (2014). Sin fines de lucro por qué la democracia necesita de las humanidades. (Vol. 1). Buenos Aires: Katz.

Pérez B, J. P., \& León, J. (2020). La nueva Gallup muestra que se acabó el 'efecto pandemia'. Recuperado el 23 de Diciembre de 2020, Recuperado de: https://lasillavacia. com/nueva-gallup-muestra-se-acabo-efecto-pandemia-78979

Ramírez, E., \& Ramírez, R. (2015). Educación en el Ejército Nacional de Colombia y el posconflicto. Criterios, 22(1), 199-214. Recuperado de: http://editorial.umariana.edu.co/ revistas/index.php/Criterios/article/view/1818

Rivera, S. (2019). Militares e identidad. Autorrepresentación y construcción de paz en el cuerpo de oficiales de las Fuerzas Militares colombianas (Vol. 1). Bogotá: Pontificia Universidad Javeriana.

Riveros, D. F. (2010). Los derechos económicos, sociales y culturales como derechos subjetivos: una visión estructural. Con comentarios de Christian Courtis. Derecho del Estado, 29-44. Recuperado de: https://revistas.uexternado.edu.co/index.php/derest/ article/view/418

Rodríguez, J. C. (2020). Algunas reflexiones sobre las percepciones de la fuerza pública en Colombia. En G. Barbosa Castillo, A. R. Ciro Gómez, \& É. Solano González, El reto de la construcción histórica del conflicto de Colombia (Vol. 9, 111-139). Universidad Externado de Colombia; Ejército Nacional de Colombia. Recuperado de: https://bdigital. uexternado.edu.co/handle/001/2786 